\title{
Yield of biliary stent cytology: Is it time to think lean?
}

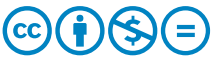

\author{
Authors \\ Douglas K. Pleskow ${ }^{1}$, Tyler M. Berzin ${ }^{1}$

\section{Institutions} \\ 1 Center for Advanced Endoscopy, Beth Israel Deaconess \\ Medical Center, Harvard Medical, Boston, \\ Massachusetts, United States \\ 2 Department of Pathology, Beth Israel Deaconess \\ Medical Center, Harvard Medical School, Boston, \\ Massachusetts, United States
}

Muthuraman Alagappan ${ }^{1}$, Natasha Darras², Lauren Yang ${ }^{1}$, Paul Vanderlaan², Meir Mizrahi ${ }^{1}$, Mandeep Sawhney',

submitted 31.5 .2018

accepted after revision 8.10 .2018

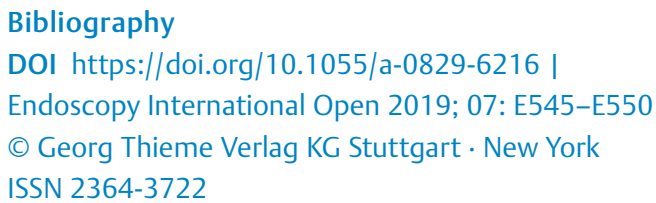

Corresponding author

Tyler M. Berzin, MD, Center for Advanced Endoscopy, Division of Gastroenterology, Beth Israel Deaconess Medical Center, 330 Brookline Avenue, Boston, MA 02215

Fax: +1-617-667-1728

tberzin@bidmc.harvard.edu

\section{ABSTRACT}

Background and study aims During evaluation of pancreaticobiliary strictures, it is common practice to send biliary stents for cytologic analysis. However, in recent years, complementary tissue acquisition techniques ranging from cholangioscopy to fine-needle biopsy have improved the ability to acquire tissue and diagnose malignancy. Data are limited on the current diagnostic yield and cost effectiveness of biliary stent analysis.

Patients and methods We performed a retrospective study of all pancreaticobiliary stents sent for analysis in a tertiary care academic medical center from June 2013 to September 2016. Patient demographics, stent information, and final diagnosis history were collected through chart review. Costs were determined using published reimbursement rates for Medicare.

Results Two hundred thirty-one stents from 175 patients were sent for cytologic analysis during the study period. Of the 62 stents obtained from patients ultimately diagnosed with malignancy, only one (1.6\%) had positive cytology for malignant cells, while the others were acellular/non-diagnostic $(2 / 62,3.2 \%)$, negative $(48 / 62,77.4 \%)$, or atypical $(11 / 62,17.7 \%)$. The sensitivity of stent cytology for diagnosis of malignancy was $1.6 \%$ (1/62). No cases were identified in which stent cytology changed clinical management. From a payer perspective, the mean estimated cost for each stent cytologic analysis is greater than $\$ 70.00$.

Conclusions While stent cytologic analysis is a common clinical practice, the diagnostic yield and cost effectiveness of the practice must be reevaluated. With the rise of newer diagnostic technologies such as digital cholangioscopy and endoscopic ultrasound-guided fine-needle biopsy, it may be time to "think lean" and acknowledge a sunset for biliary stent cytology.

\section{Introduction}

Accurate and early diagnosis of pancreaticobiliary malignancy is a core goal of modern therapeutic endoscopy [1]. There are multiple tissue-based diagnostic modalities for evaluation of pancreaticobiliary malignancy, including cytology brushing and fluorescence in situ hybridization (FISH), biliary stent cytology, per-oral cholangioscopy with directed biopsies, and endoscopic ultrasound (EUS)-guided fine-needle aspiration (FNA) and biopsy (FNB) [1 -4]. Although the diagnostic yield of these techniques is variable, recent studies have reported diagnostic accuracies as high as $96 \%$ for cholangioscopy and sensitivities approaching $80 \%$ to $90 \%$ for EUS-FNA and EUS-FNB [4-9]. These newer technologies are leading the way toward highyield and cost-effective diagnosis of pancreaticobiliary malignancy.

Biliary stents, which are routinely placed during endoscopic retrograde cholangiopancreatography (ERCP) for treatment of biliary strictures, are frequently sent for cytologic analysis upon removal if there is diagnostic uncertainty and if malignancy has not been conclusively ruled out. Early studies reported stent cytology sensitivities as high as $78.6 \%$ for diagnosis of malignancy [10-11]. However, a later study in 2003 demonstrated much lower sensitivity, ranging between $11 \%$ to $16 \%$ 
[12]. More recent evidence supporting a continued role for stent cytologic analysis is lacking.

With the introduction of newer, highly sensitive and specific diagnostic modalities for diagnosing pancreaticobiliary malignancy, it is important to consider whether older diagnostic options such as stent cytology can be discarded due to their low yield, poor practicality, or cost ineffectiveness. The primary aim of this study was to determine the current diagnostic yield of biliary stent cytology, with a secondary aim of estimating the cost of this approach in comparison to other diagnostic methods.

\section{Patients and methods}

\section{ERCP for biliary strictures}

Patients who had a biliary or pancreatic stent removed and sent for cytologic analysis between June 2013 and September 2016 at our tertiary care academic medical center were retrospectively reviewed.

Patients were referred to our center for ERCP for treatment and evaluation of known or suspected biliary strictures. All procedures were performed in a single academic referral center. ERCP was performed by a dedicated therapeutic endoscopy team using standard technique. Brush cytology was routinely performed in all cases. The decision to additionally perform direct cholangioscopy or EUS-FNA/FNB during the same session or at a later time was at the discretion of the endoscopist. After stent placement, a follow-up ERCP was performed for stent removal, reevaluation, or exchange (to metal stent) if malignancy had been confirmed. At the time of follow-up ERCP, the removed biliary stent was only sent for cytology if a diagnosis had not already been confirmed during prior evaluation. In these cases, the stent was typically removed with a snare or forceps, placed immediately in a vial of the methanol-based fixative Cytolyt (Hologic, Marlborough, Massachusetts, United States), and sent to the cytology laboratory for processing and evaluation.

\section{Cytology analysis}

Upon receipt in the cytology laboratory, the Cytolyt vial was vortexed to disaggregate any cellular material adherent to the stent, the stent removed, and the Cytolyt fluid used to prepare a single ThinPrep monolayer cytology slide using a ThinPrep 2000 processor per standard manufacturer instructions (Hologic, Marlborough, Massachusetts, United States). The ThinPrep slide was stained using the Papanicolaou method, screened by a cytotechnologist and a cytopathology fellow, with final review performed by one of seven board-certified cytopathologists in our department. Diagnostic reporting for these specimens followed standard cytology categorization: non-diagnostic (i.e. a cytology specimen that provides no diagnostic or useful information about the lesion sampled), negative for malignant cells, atypical (i.e. cytologic changes that are more likely than not to be benign), suspicious for malignant cells (i. e. cytologic changes that are more likely than not to be malignant), and positive for malignant cells.

\section{Study design and analysis}

Cases were identified by searching the cytology laboratory information system for accessioning source codes used for biliary stents. Patients were included in this study if they were aged 18 or older and had a biliary or pancreatic stent removed and sent for cytologic analysis between June 2013 and September 2016. Pancreatic stents were excluded due to low number of cases. Patients with incomplete data were excluded from this study. Demographics, stent placement and removal date, stent type, date of last follow-up, and final diagnoses were collected manually through chart review. The results of the cytology analysis were collected through manual chart review in collaboration with a pathologist. The method of final diagnosis was also recorded, with options including surgical biopsy, ERCP brushing, EUS-FNA or -FNB, or serial imaging, among others. Test characteristics for biliary stent cytology including sensitivity, specificity, positive predictive value (PPV), and negative predictive value (NPV) were calculated.

Technical and professional costs for biliary stent cytology were determined using Medicare reimbursement rates in 2016 [13-14]. CPT code 88112 for cytopathology (selective cellular enhancement technique with interpretation) was used to query the Medicare Physician Fee Schedule Search. CPT code 43260 was used for diagnostic ERCP with collection of specimen by brushing, and CPT code 43238 was used for EUS with FNA/FNB [13]. Physician and facility fees were estimated based on the 2016 Medicare national average payment [14].

This study was approved by the Institutional Review Board at the Beth Israel Deaconess Medical Center in Boston, Massachusetts.

\section{Results}

Between June 2013 and September 2016, a total of 231 biliary stents, from 175 patients, were received for cytology analysis by our cytology department. Of these 175 patients, 105 were male and 70 were female. The mean age of patients at time of stent removal was 65.8 years (range $24-94$ years). Of the 231 stents, 210 were plastic and 21 were metal. All stents were biliary. Stent duration information was available for 182 stents. Information was not available for the remaining stents because they were placed at an outside facility where the stent placement date was not clearly recorded. The mean number of days between stent placement and removal (i.e. stent duration) was 66.3 days (median 42 days, standard deviation 69.1 days, range 2-621 days).

Of the 231 stents sent for cytology, 10 (4.3\%) were acellular (non-diagnostic), 191 (82.7\%) were negative, 29 (12.6\%) were atypical, and one $(0.4 \%)$ was positive for malignancy ( $\triangleright$ Table 1). The final diagnoses of the patients for each removed stent were recorded, and the most common diagnoses were malignancy (62 stents, $26.8 \%$ ), chronic pancreatitis (35 stents, $15.2 \%$ ), and other benign biliary/pancreatic etiologies (87 stents, $37.7 \%$ ) ( $\triangleright$ Table 1 ). Of these 231 stents, one was in a patient with a diagnosis of both chronic pancreatitis and malignancy, and three were in patients with a diagnosis of both 
- Table 1 Stent cytology results by diagnosis.

\begin{tabular}{|c|c|c|c|c|}
\hline Diagnosis & Acellular/non-diagnostic & Negative & Atypical & Positive for malignant cells \\
\hline Chronic pancreatitis & $0.0 \%(0 / 35)$ & $94.3 \%(33 / 35)$ & $5.7 \%(2 / 35)$ & $0.0 \%(0 / 35)$ \\
\hline Primary sclerosing cholangitis & $3.9 \%(1 / 26)$ & $92.3 \%(24 / 26)$ & $3.9 \%(1 / 26)$ & $0.0 \%(0 / 26)$ \\
\hline Autoimmune pancreatitis & $0.0 \%(0 / 17)$ & $76.5 \%(13 / 17)$ & $23.5 \%(4 / 17)$ & $0.0 \%(0 / 17)$ \\
\hline Benign & $6.9 \%(6 / 87)$ & $83.9 \%(73 / 87)$ & $9.2 \%(8 / 87)$ & $0.0 \%(0 / 87)$ \\
\hline Malignancy & $3.2 \%(2 / 62)$ & $77.4 \%(48 / 62)$ & $17.7 \%(11 / 62)$ & $1.6 \%(1 / 62)$ \\
\hline No diagnosis & $12.5 \%(1 / 8)$ & $50.0 \%(4 / 8)$ & $37.5 \%(3 / 8)$ & $0.0 \%(0 / 8)$ \\
\hline
\end{tabular}

- Table 2 Yield of stent cytology for diagnosis of malignancy.

\begin{tabular}{|l|l|l|l|}
\hline & Final diagnosis & Other \\
\hline Stent cytology & Malignancy & 0 \\
\hline & Positive for malignant cells & 1 & 169 \\
\hline Sensitivity of stent cytology for diagnosis of malignancy was $1.61 \%(1 / 62)$ and the negative predictive value was $73.5 \%(169 / 230)$.
\end{tabular}

primary sclerosing cholangitis and malignancy ( $>$ Table 1 ). The benign etiologies included inflammation from choledocholithiasis, ischemic/anastomotic strictures, and strictures of unknown etiology (which remained stable or resolved upon follow up). Average duration of clinical follow-up for patients with benign etiologies was 35.3 weeks (standard deviation 47.4 weeks).

Among the 62 stents in patients ultimately diagnosed with malignancy, two were acellular/non-diagnostic (3.2\%), 48 were negative for malignancy (77.4\%), 11 were atypical (17.7\%), and one was positive for malignancy $(1.6 \%)$. The overall yield was $0.4 \%$ (1/231). Sensitivity of stent cytology for diagnosis of any malignancy was $1.6 \%$ and specificity was $100 \%$. PPV was $100 \%$ and the NPV was $73.5 \%$ ( $\downarrow$ Table 2 ). There were no false positives and 48 false negatives. Of the one positive stent cytology, the brushings from the same ERCP were also positive. The PPV and specificity of atypical cells on cytology for malignancy were $37.9 \%(11 / 29)$ and $89.3 \%$ (151/169), respectively.

Malignancy was diagnosed in 49 patients. Pathology from another source was used to make the diagnosis in 46 of these cases (93.9\% of malignant diagnoses). Malignancy was confirmed through surgical or imaging-guided percutaneous biopsies in 19 cases, bile duct brushings alone in eight cases, EUSFNA alone in nine cases, standard endoscopy cold forceps biopsies in four cases, EUS-FNB in two cases, and more than one ERCP/EUS-based tissue acquisition method in four cases. Three patients were presumed to have a malignant diagnosis based on clear imaging and clinical features. One patient was treated for malignancy with a chemotherapy regimen at another institution and two underwent work-up for cholangiocarcinomaprotocol liver transplant. The two most common malignancy diagnoses were cholangiocarcinoma (17/49, 34.7\%) and pancreatic adenocarcinoma (16/49, 32.7\%) ( $>$ Table 3$)$.

Once an ERCP is performed for stent removal, the incremental cost of biliary stent cytology is $\$ 29.52$ for technical processing (CPT 88112) and $\$ 40.68$ for professional review (CPT 88112 ), totaling $\$ 70.20$ additional cost to the payer per procedure [13]. If ERCP brushing cytology is performed, the incremental costs are approximately $\$ 125.00$ for the cytology brush catheter and $\$ 70.20$ for cytologic analysis (CPT 88112), totaling $\$ 195.20$ additional cost to the payer per procedure [13]. If an EUS-FNA/FNB is performed to assist in diagnosis, the incremental costs are approximately $\$ 250.00$ for the physician fee (CPT 43238), approximately $\$ 1088.00$ for the facility fee (CPT 43238), approximately $\$ 375.00$ for the EUS FNB device, and $\$ 70.20$ for cytologic analysis (CPT 88112), totaling $\$ 1783.20$ additional cost to the payer per procedure [13-14].

\section{Discussion}

Pancreaticobiliary malignancies are aggressive diseases associated with high mortality [15]. Early and accurate diagnosis has been a central goal of pancreaticobiliary endoscopy, and our evolving armamentarium of tools ranging from cholangioscopy to EUS-FNA/FNB has improved our ability to provide highly accurate tissue diagnoses for this patient population [4 - 8, $16-$ 17]. Biliary stent cytology has "hung on" in many practices because it is believed to provide reasonable diagnostic value, with reported sensitivities ranging from $11 \%$ to $78 \%$ in prior studies of relatively small sample size, and because gastroenterologists may not want to discard potentially valuable samples if a stent has been retrieved in a patient where diagnostic uncertainty remains [10-12]. However, given the presence of multiple new 
- Table 3 Malignancy diagnoses by type and number of patients.

\begin{tabular}{|l|c|c|}
\hline Cancer Diagnosis & $\begin{array}{l}\text { Number of } \\
\text { patients }\end{array}$ & $\begin{array}{l}\text { Percentage } \\
\text { (\%) }\end{array}$ \\
\hline Cholangiocarcinoma & 17 & $34.7 \%$ \\
\hline Pancreatic adenocarcinoma & 16 & $32.7 \%$ \\
\hline Pancreatobiliary cancer & 6 & $12.2 \%$ \\
\hline Ampullary cancer & 3 & $6.1 \%$ \\
\hline Hepatocellular carcinoma & 2 & $4.1 \%$ \\
\hline Gallbladder cancer & 1 & $2.0 \%$ \\
\hline Melanoma & 1 & $2.0 \%$ \\
\hline Neuroendocrine tumor & 1 & $2.0 \%$ \\
\hline Diffuse large B-cell lymphoma & 1 & $2.0 \%$ \\
\hline Cancer of unknown primary & 1 & $2.0 \%$ \\
\hline $\begin{array}{l}\text { The most common malignancies were cholangiocarcinoma and pancreatic } \\
\text { adenocarcinoma. }\end{array}$ & & \\
\hline
\end{tabular}

and effective diagnostic approaches, it is important to consider whether we should stop sending biliary stents for cytologic analysis.

Our study is the largest study to evaluate the diagnostic yield of biliary stent cytology, and the first to do so in the "modern" era of pancreaticobiliary endoscopy. We found that the sensitivity of stent cytology was only $1.6 \%$ for diagnosis of any malignancy. Of all 231 stents sent for cytology, only one was positive for malignant cells $(0.4 \%)$, and in that patient, the brushings from the same ERCP were also positive for malignancy. "Atypical" stent cytology provided a PPV of only $37.9 \%$.

It is notable that our reported sensitivity is significantly lower than what has been previously published, for which there are at least two possible explanations. One possible explanation for the discordance in findings is a difference in sample size. The initial study in 1989 by Leung et al. reported a 78.6\% sensitivity for diagnosis of malignancy after examination of stents, but that study included only 14 patients [11]. In a more recent study by Devereaux et al. in 2003 , a sensitivity of up to $16 \%$ was reported depending on how atypia was interpreted, but this study only included 126 stents from 101 patients [12]. We suspect that a second reason for diminishing sensitivity of stent cytology is that the very high yield of modern EUS-FNB, cholangioscopy, and related techniques likely allows clinicians to diagnose malignancy earlier and more accurately. As a result, at the time of follow-up ERCP for stent removal or exchange, the remaining patients with biliary strictures of unknown cause are more likely to have benign etiologies and/or undiagnosed malignancies which have not yielded adequate tissue through multiple sampling techniques. We suspect this pattern is likely reflective of the current era of pancreaticobiliary endoscopy.

In comparison to stent cytology, several other diagnostic techniques have demonstrated far higher sensitivities, with ERCP brush cytology generally approaching greater than $60 \%$ sensitivity and EUS FNA/FNB and cholangioscopy exceeding

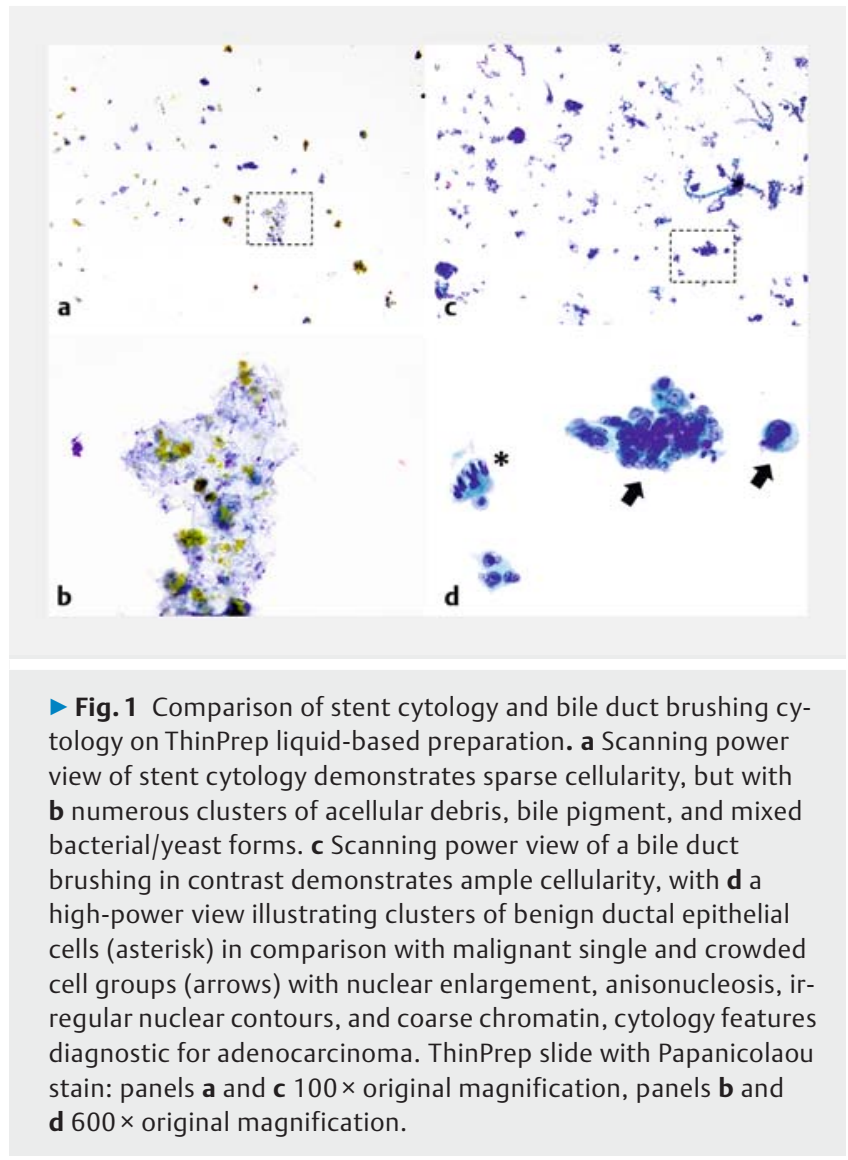

$90 \%$ sensitivity in many studies $[1-9,18]$. Slide preparations from ERCP brushings have more cellularity than slide preparations from biliary stents ( $\vee$ Fig. 1 ), so it is unlikely that stent cytology will be positive when the ERCP brushings cytology is not. As clinicians routinely send ERCP brushings for cytology and only occasionally "add-on" stent cytology, there appears to be little incremental value in this practice. In our study, the only patient with positive stent cytology also had a positive ERCP brushing cytology for malignancy. No cases were identified in our study where positive stent cytology changed clinical management. Further, the NPV of stent cytology in our study was $73.5 \%$, which implies that a negative result is not enough to rule out the presence of cancer. As more effective and sometimes equally low-cost (e.g. ERCP brushings) alternatives exist, it may be important to move away from stent cytology as a diagnostic approach.

Despite the poor sensitivity and low NPV, some practitioners may choose to continue sending stent cytology due to the low perceived cost. However, the incremental cost of stent cytology is $\$ 70.20$ for a sensitivity of only $1.6 \%$. In comparison, the incremental cost of ERCP brushing cytology is $\$ 195.20$ for a sensitivity of $65 \%$, and the incremental cost of EUS-FNA/FNB is $\$ 1783.20$ for a sensitivity of $84 \%$ [18-19]. Approximately 450,000 ERCPs are performed annually in the United States, and we estimate that stents may be removed and sent for cytology in up to $5 \%$ of ERCPs based on our institutional experience [20]. With these estimates, eliminating the practice of stent cy- 
tology would result in a theoretical cost savings to the healthcare system of more than \$1.57 million per year. Although technical and professional costs per stent cytology seem low, they represent a real, incremental cost to the healthcare system and provide very little value in return, especially when more sensitive diagnostic modalities are employed during the same procedure.

Another advantage to discarding stent cytology as a diagnostic option for malignancy is embracing de-innovation, the process of eliminating "entrenched and often costly practices that previously made sense but that, because of new evidence or competing approaches, have lost their value" [21]. Clinical practice has become increasingly cluttered by an expanding array of tests and services. By discarding low-value care, we can aim to reduce healthcare costs and open the field for new, more effective innovations.

There are several limitations to our study. This study was retrospective and other diagnostic methods (cholangioscopy, EUS-FNB) have been evolving and in increasing use during the study period. In addition, this study was performed at a single institution, and the diagnostic yield of stent cytology may vary based on institutional practice, depending on multiple factors, including the patient population and how other advanced tissue acquisition techniques are employed during the diagnostic workup. Further, there may be variability among cytopathologists with respect to their institutional thresholds for diagnosing a specimen as "atypical" or "positive for malignancy." Cytopathologists with a higher threshold for diagnosing atypia or malignancy will have a lower observed sensitivity of stent cytology.

\section{Conclusion}

In conclusion, there are now several highly sensitive and specific diagnostic options for pancreaticobiliary malignancies, such as cholangioscopy and EUS-FNB. Although biliary stent cytology has previously been reported to have high sensitivities for diagnosis of malignancy, our study found the sensitivity to be less than $2 \%$, perhaps due to evolving clinical patterns and earlier diagnoses of malignancy. The availability of better diagnostic modalities, low sensitivity, poor NPV, and incremental cost make stent cytology an undesirable option. In the spirit of deinnovation and high-value care, we favor abandoning this practice in patients with strictures of low pre-test probability for malignancy.

\section{Competing interests}

Drs. Berzin and Pleskow are consultants for Boston Scientific and Medtronic.

\section{References}

[1] Bledsoe JR, Shinagare SA, Deshpande V. Difficult Diagnostic Problems in Pancreatobiliary Neoplasia. Arch Pathol Lab Med 2015; 139: 848 857

[2] Burnett AS, Bailey J, Oliver JB et al. Sensitivity of alternative testing for pancreaticobiliary cancer: a 10-y review of the literature. J Surg Res 2014; 190: $535-547$

[3] Kalaitzakis E, Levy M, Kamisawa T et al. Endoscopic retrograde cholangiography does not reliably distinguish IgG4-associated cholangitis from primary sclerosing cholangitis or cholangiocarcinoma. Clin Gastroenterol Hepatol 2011; 9: 800-803 e2

[4] Pereira P, Peixoto A, Andrade P et al. Peroral cholangiopancreatoscopy with the $\operatorname{SpyGlass}(R)$ system: what do we know 10 years later. J Gastrointestin Liver Dis 2017; 26: 165-170

[5] De Moura DTH, Moura EGH, Bernardo WM et al. Endoscopic retrograde cholangiopancreatography versus endoscopic ultrasound for tissue diagnosis of malignant biliary stricture: Systematic review and meta-analysis. Endosc Ultrasound 2018; 7: 10 - 19

[6] Mohamadnejad M, Mullady D, Early DS et al. Increasing number of passes beyond 4 does not increase sensitivity of detection of pancreatic malignancy by endoscopic ultrasound-guided fine-needle aspiration. Clin Gastroenterol Hepatol 2017; 15: 1071 - 1078 e2

[7] Banafea O, Mghanga FP, Zhao J et al. Endoscopic ultrasonography with fine-needle aspiration for histological diagnosis of solid pancreatic masses: a meta-analysis of diagnostic accuracy studies. BMC Gastroenterol 2016; 16: 108

[8] DiMaio CJ, Kolb JM, Benias PC et al. Initial experience with a novel EUSguided core biopsy needle (SharkCore): results of a large North American multicenter study. Endosc Int Open 2016; 4: E974-989

[9] Cheng B, Zhang Y, Chen Q et al. Analysis of fine-needle biopsy versus fine-needle aspiration in diagnosis of pancreatic and abdominal masses: a prospective, multicenter, randomized controlled trial. Clin Gastroenterol Hepatol 2018; 16: 1314-1321

[10] Foutch PG, Kerr DM, Harlan JR et al. A prospective, controlled analysis of endoscopic cytotechniques for diagnosis of malignant biliary strictures. Am J Gastroenterol 1991; 86: 577-580

[11] Leung JW, Sung JY, Chung SC et al. Endoscopic scraping biopsy of malignant biliary strictures. Gastrointest Endosc 1989; 35: 65-66

[12] Devereaux BM, Fogel EL, Bucksot L et al. Clinical utility of stent cytology for the diagnosis of pancreaticobiliary neoplasms. Am J Gastroenterol 2003; 98: 1028-1031

[13] Physician Fee Schedule (MPFS). Centers for Medicare \& Medicaid Services; Available at: https://www.cms.gov/medicare/medicare-feefor-service-payment/physicianfeesched/ Accessed January 4, 2019

[14] Procedural Reimbursement Guide for Endoscopy 2016. Boston Scientific; Available at: https://www.bostonscientific.com/content/dam/ bostonscientific/Reimbursement/Gastroenterology/2016/2016_Procedural_Reimbursement_Guide_Endoscopy.pdf/ Accessed January 4, 2019

[15] Survival statistics for bile duct cancers. American Cancer Society; Available at: https://www.cancer.org/cancer/bile-duct-cancer/detection-diagnosis-staging/survival-by-stage.html Accessed January 4, 2019

[16] Fritscher-Ravens A, Broering DC, Knoefel WT et al. EUS-guided fineneedle aspiration of suspected hilar cholangiocarcinoma in potentially operable patients with negative brush cytology. Am J Gastroenterol 2004; 99: 45 - 51

[17] Jailwala J, Fogel EL, Sherman S et al. Triple-tissue sampling at ERCP in malignant biliary obstruction. Gastrointest Endosc 2000; 51: $383-$ 390

[18] Mehmood S, Loya A, Yusuf MA. Biliary brush cytology revisited. Acta Cytol 2016; 60: $167-172$ 
[19] Onda S, Ogura T, Kurisu Y et al. EUS-guided FNA for biliary disease as first-line modality to obtain histological evidence. Therap Adv Gastroenterol 2016; 9: $302-312$

[20] Kozarek RA. The future of ERCP. Endosc Int Open 2017; 5: E272 -E274
[21] Ubel PA, Asch DA. Creating value in health by understanding and overcoming resistance to de-innovation. Health Affairs 2015; 34: $239-244$ 